Slavica

bruxellensia

\section{Slavica bruxellensia}

Revue polyphonique de littérature, culture et histoire

slaves

$11 \mid 2015$

Littérature et philosophie

\title{
Traduction du récit « Maudits médicaments!» (Prekleta zdravila!) de Miha Mazzini
}

Traduction du slovène

\section{Stéphane Bultel, Meta Klinar et Cécile Bocianowski}

\section{OpenEdition}

Journals

Édition électronique

URL : http://journals.openedition.org/slavica/1683

DOI : 10.4000/slavica.1683

ISSN : 2034-6395

Éditeur

Université libre de Bruxelles - ULB

Référence électronique

Stéphane Bultel, Meta Klinar et Cécile Bocianowski, «Traduction du récit « Maudits médicaments! » (Prekleta zdravila!) de Miha Mazzini », Slavica bruxellensia [En ligne], 11 | 2015, mis en ligne le 15 février 2015, consulté le 01 mai 2019. URL : http://journals.openedition.org/slavica/1683 ; DOI : 10.4000/ slavica. 1683

Ce document a été généré automatiquement le 1 mai 2019.

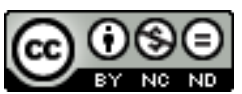

Les contenus de Slavica bruxellensia sont mis à disposition selon les termes de la Licence Creative Commons Attribution - Pas d'Utilisation Commerciale - Pas de Modification 3.0 France. 


\section{Traduction du récit « Maudits} médicaments!»(Prekleta zdravila!) de Miha Mazzini

Traduction du slovène

Stéphane Bultel, Meta Klinar et Cécile Bocianowski

\section{Présentation}


«Maudits médicaments! »a paru dans le recueil d'histoires courtes Duhovi (Les Esprits) ${ }^{1}$. Cette nouvelle traite l'un des thèmes les plus fréquents chez Miha Mazzini : la relation fatale entre les parents et les enfants. L'auteur décrit, sous la forme d'un monologue, la vie d'une mère célibataire qui consacre sa vie aux soins de sa fille unique gravement malade.

Dans le style d'écriture d'un scénariste expérimenté, Mazzini développe l'histoire au fil des phrases, où chaque mot tient sa juste valeur, et maintient l'intérêt du lecteur jusqu'à la fin dramatique.

Pour plus d'information sur Miha Mazzini, nous vous invitons à le découvrir dans l'entretien mené par Meta Klinar et Dorota Walczak-Delanois disponible dans ce numéro.

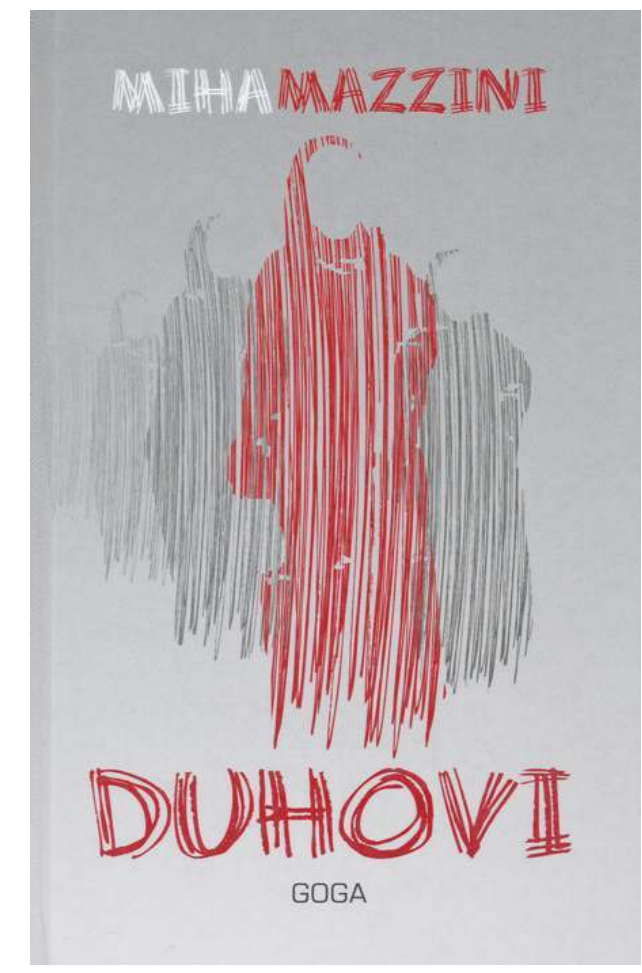

\section{Traduction}

2 Je t'en prie, écoute ma douleur. J'ai eu une fille, je m'en suis occupée, enfin cela a duré quatre ans, deux mois et vingt-six jours, comme ça, immobile, je la levais avec mes faibles mains, je la retournais, la pommadais, je lui changeais sa couche, je la déplaçais dans la poussette, je l'emmenais jusqu'à la fenêtre, jusqu'à l'air frais, jusqu'à la lumière, ces yeux qui sont les siens, immobiles comme des billes de laine quand je les regardais de côté, la peau toujours plus blanche, transparente, des veinules, ma fille. Ma fille. Ensuite est arrivé ce médecin, nouveau, jeune, il m'a paru suspect, trop plein de confiance en lui, trop enthousiaste, est-ce que cela convient à un tel malade? Je devais signer une autorisation pour un nouveau médicament. Il tenait un stylo à la main. Bien sûr, j'ai signé, qui ne l'aurait fait? L'amour maternel est d'une confiance aveugle. Il est vrai que d'autres l'avaient précédé, ils avaient essayé, mais rien, ni mieux ni pire, rien. Comment aurais-je pu refuser, dis-moi, comment? Je ne pouvais pas savoir que...

Il lui a fait une injection, rien, d'abord rien. La fenêtre était ouverte, le printemps, 13 mars, ça souffle, me suis-je dit, ça souffle, ah non, non, elle s'est mise à trembler, ses bras, ses jambes, combien de fois les ai-je massés, pour éviter les escarres, son corps, osseux, seulement des os, elle a tremblé, claqué, ma fille, au secours, au secours, je criais, mais lui... il regardait seulement... et puis... ma fille, ma fille.

Maudits médicaments! Ils ont pris ma fille !

Quand elle était petite, je l'observais pendant qu'elle dormait. Les enfants sont des anges, quand ils dorment. Ensuite j'arrangeais sa couverture, tout doucement, pour qu'elle ne se réveille pas, plus pour justifier ma présence. Quand ils l'ont renvoyée de l'hôpital, je l'ai regardée aussi comme ça et je n'ai pas compris dans un premier temps que, maintenant, elle serait comme ça, tu comprends. Pour toujours immobile, dépendante de moi, comme ça. Alors j'arrangeais sa couverture, je suivais leurs consignes, ce que me montrait l'infirmière à domicile, $\mathrm{j}$ 'ai tout fait, mais quand l'infirmière partait, je marchais sur la 
pointe des pieds, ma fille dormait, je la regardais, puis j'arrangeais le drap.

Et je lui parlais, on m'a dit dans la salle d'attente qu'ils savent ce qui se passe, ils reconnaissent les voix, je lui en ai tellement raconté !

Maintenant je reste debout près de son lit vide et je sais que jamais plus, tu comprends le sens de ce que je dis, que jamais plus elle ne s'allongera sur ce lit. J'arrange le drap, j'ajuste la couverture. Il n'y a personne sous la couverture, je caresse le tissu pour que les bords soient parfaits.

Je pleure. Maudits médicaments, maudits médicaments !

Elle était partie en excursion scolaire et avait pris froid. Je lui avais dit qu'elle n'était pas assez habillée, elle ne m'a pas écoutée. Elle approchait de la puberté, elle était têtue. Les médecins disaient que c'était une inflammation du cerveau, que ce n'était pas un rhume, mais qu'est-ce qu'ils en savent, comme celui qui a... je ne veux surtout pas me rappeler de lui, tu comprends, avec ce qu'il m'a fait ! Elle était rentrée de l'excursion avec de la fièvre, la nuit elle a déliré, le matin j'ai voulu l'emmener chez le médecin, elle ne m'a pas reconnue. Pas du tout. Elle a regardé dans ma direction et rien, tu comprends, rien. J'ai sollicité le voisin. L'hôpital, un mois près d'elle, et elle ne s'est plus réveillée.

Je l'ai nourrie, j'ai tout appris, l'infirmière à domicile n'a plus été nécessaire. J'ai abandonné mon travail, mes amies, tu sais, elles n'étaient pas nombreuses, je disais que je n'avais plus le temps, et bientôt elles ont cessé de venir, je n'avais pas d'homme, ils ne m'intéressent pas depuis que je sais comment ils sont, d'après mes propres expériences, tu sais, je restais à la maison, je la surveillais. Pour nos besoins, l'aide sociale suffisait, nous étions modestes, peu exigeantes. Parfois, dans le temps, avant la maladie, elle me reprochait d'avoir un salaire trop bas en tant que femme de ménage, et que ses camarades de classe et ses amies étaient vêtues de marques connues, et elle pas, et ça à cause de moi, mais quand elle est tombée malade, où sont passées toutes ces copines, où ? Elles sont venues la voir une fois à l'hôpital, ensuite plus jamais. Nous sommes restées seules, seules.

Mais on ne s'ennuyait pas, jamais. Quel travail il y avait avec elle! Encore maintenant j'ai mal au dos quand j'y pense, comment je l'ai levée, elle était une jeune fille grande pour son âge, ensuite elle a encore grandi, mais plus lentement, plus lentement. Elle devenait adulte, au lit. Tout était si lent. Elle changeait doucement, son visage s'allongeait quel que soit son régime, les cheveux devenaient plus clairs, si beaux, combien de fois les ai-je peignés près de la fenêtre, je préférais près de la fenêtre. Quatre ans, deux mois et vingtsix jours, jusqu'à ce maudit médecin et ses médicaments !

Près de la fenêtre... C'était si beau, toujours. Dis-moi, est-ce que c'est possible, quelque chose qui soit beau à chaque fois ? Une fois, deux fois... mais à chaque fois, à chaque fois. Surtout en été. Tu sais, en été dans cette rue, il y a une lumière vraiment spéciale. Et ses cheveux étaient dans cette lumière... Tu le sais, tu le sais. Quelles plaisanteries nous faisions, les beaux jours... Je la mettais à la fenêtre, bien habillée mais seulement en haut, la partie que l'on voyait de la rue, je lui mettais ses lunettes de soleil, je la peignais bien, je mettais un miroir sur l'appui de fenêtre et je me cachais, assise sur l'escabeau parce que mes jambes me font mal, je ne peux plus m'agenouiller comme avant, et nous attendions. Tôt ou tard, un garçon passait, peut-être un facteur, un étudiant qui distribuait le courrier l'été, je le sentais venir, je le voyais dans le miroir et je levais sa main gauche, je saisissais ma fille sous l'occiput et lui faisait hocher la tête. Le petit ami s'arrêtait, lui aussi hochait la tête. Il souriait et commençait à parler. Je faisais acquiescer ma fille de la tête ou faire signe que non avec la main, en fonction de ce qu'il disait. Il nous invitait à sortir, aller au cinéma, je me retenais à peine d'éclater de rire, j'étais secouée de rire, je 
remuais le fauteuil roulant, pour sûr ça se voyait qu'elle aussi riait. Dans le miroir, je voyais leurs visages déconcertés, ils se demandaient ce qu'ils avaient dit de travers, ce qui se passait, comme ils étaient déconcertés, tu sais comment sont les hommes quand ils n'ont pas ce qu'ils veulent. Comme nous nous vengions d'eux, comme nous nous amusions!

Mais ensuite...

Ce médecin et son médicament!

Je te le dis entre nous: je ne m'attendais pas à ce que ça aille de travers. Parce qu'ils avaient déjà essayé tant d'autres fois. Mais quand j'ai vu comme elle tremblait, j'ai seulement ouvert la bouche, je n'ai rien senti, pas le temps. Sur le coup, je n'ai pas pu croire que c'en était fini.

La fin de tout.

Quand tu as un tel enfant, tu t'enroules autour de lui comme une peau protectrice. Puis quand il n'est plus là, tu restes vide, ils ne te rempliront pas d'une nouvelle chair et de nouveaux os.

Elle s'est levée. Sur les baguettes qu'elle avait encore à la place des jambes, elle restait debout. Elle se balançait, le vent, la fenêtre ouverte, elle se berçait, elle tombait, le médecin la rattrapait. Il éclatait de rire, le salaud, il riait.

Il travaille, travaille, il pousse un cri et elle s'appuie sur lui, elle pousse sur ses bras, sa tête se tourne lentement, tant qu'elle ne me voit pas.

Dans ses yeux, j'ai vu qu'elle m'avait reconnue.

Il y a déjà dix ans de cela. Et voilà qu'elle vient parfois, une fois par an, au repas de Noël, elle vient en famille, comme avant la maladie, têtue, obstinée, je regarde ses enfants et son mari, c'est seulement pour ça qu'elle les emmène, pour se prévaloir de ce que je n'ai pas, j'espère qu'ils cesseront de venir et qu'ils m'enverront seulement leurs vœux que je laisserai dans la boîte aux lettres.

Maudits, maudits médicaments !

\section{NOTES}

1. Ed. Goga, Ljubljana, 2010

\section{INDEX}

Index chronologique : XXe siècle

Mots-clés : littérature slovène

Index géographique : Slovénie 


\section{AUTEURS}

\section{META KLINAR}

Lectrice de slovène, Université Libre de Bruxelles

\section{CÉCILE BOCIANOWSKI}

Doctorante en Littérature comparée à l'Université Paris IV-Sorbonne (France) ; assistante à

l'Université Libre de Bruxelles (Belgique) ; membre du comité de rédaction de Slavica Bruxellensia 